\title{
Presencia de micorrizas en orquídeas de un jardín botánico neotropical
}

\author{
Marta Rivas ${ }^{1}$, Jorge Warner ${ }^{2}$, Mariela Bermúdez ${ }^{3}$ \\ Centro de Investigación General, Universidad Estatal a Distancia, E.mail: mrivas@arenal.uned.ac.cr \\ Jardín Botánico Lankester, Universidad de Costa Rica, Apdo. 1031-7050 Cartago, Costa Rica. \\ Museo Nacional de Costa Rica, San José, Costa Rica.
}

Recibido 20-II-1997. Corregido 10-XI-1997. Aceptado 14-I-1998

\begin{abstract}
Mycorrhizal occurrence was tested in roots of 24 terrestrial and epiphytic orchid species growing at the Lankester Botanical Gardens (Cartago, Costa Rica). Standard staining and light microscopy were used to search for pelotons. Roots in contact with the substrate harbor extensive mycorrhyzal fungi. There may be a wide distribution and density of the fungal flora in the secondary forests of the site, this could explain the high natural regeneration of orchids on the garden.
\end{abstract}

Key words: Mycorrhizae, Orchidaceae, cultivated plants, occurence.

El término micorriza se refiere a la relación simbiótica entre un hongo y las raíces de una planta vascular. En la mayoría de las asociaciones micorrícicas, el hongo que coloniza la raíz se beneficia con los productos de la fotosíntesis mientras que la planta incrementa la absorción de agua y nutrientes, principalmente fósforo (Harley y Smith 1983, citados por Lesica y Antibus 1990). En algunos casos las plantas se benefician digiriendo el hongo y aprovechando sus productos.

De acuerdo con la localización de las hifas con respecto a las células, las micorrizas se clasifican en ectomicorrizas y endomicorrizas. Las hifas de las ectomicorrizas forman un manto alrededor de las raíces sin penetrar las células. En las endomicorrizas el hongo crece dentro de las células corticales de la raíz y forma estructuras características (Currah 1991). Existen dos tipos de endomicorrizas, el grupo más común se dis- tingue por presentar hifas aseptadas, vesículas y estructuras ramificadas que le confieren el nombre de micorrizas vesículo-arbusculares. Estos hongos son probablemente táxones primitivos relacionados con Zygomycota y sus aliados. Aproximadamente $90 \%$ de las dicotiledóneas y la mayoría de las monocotiledóneas presentan este tipo de micorriza (Currah 1991).

El segundo grupo está constituido por hongos con hifas septadas que invaden las células de la raíz sin romper la membrana plasmática y crecen dentro de la célula formando estructuras globosas conocidas como pelotones (Richardson et al. 1993). Sus cultivos puros han dado como resultado hongos basidiomicetes y hongos imperfectos, especialmente miembros del complejo Rhizoctonia. Este tipo de micorriza es menos común y se encuentra solamente en las familias Ericaceae y Orchidaceae (Hadley 1982). 
A pesar de que la mayoría de los miembros de la familia Orchidaceae son epífitas tropicales y subtropicales, la presencia de micorrizas ha sido poco investigada. El grupo mejor estudiado es el de las orquídeas terrestres, particularmente las especies nativas de Norteamérica (Currah et al. 1987), Europa (Marchisio et al. 1985), parte de Asia (Hadley y Williamson 1972, Hadley 1985, Tsutsui y Tomita 1986, Uetake et al. 1992,) y Australia (Warcup y Talbot 1967, 1971, 1980, Warcup 1981, 1985, Ramsay et al. 1986).

La mayoría de las especies de zonas templadas están siempre infectadas con micorrizas, pero los estudios hechos con orquídeas tropicales han mostrado grados variables de asociación (Alexander 1987). Algunos autores han escrito que la infección de micorrizas en zonas tropicales es esporádica o menos común que en orquídeas terrestres de zonas templadas (Hadley y Williamson 1972, Hadley 1986, Lesica y Antibus 1990) y otros han informado lo contrario (Benzing y Friedman 1981, Benzing 1982, Alexander 1987, Bermudes y Benzing 1989, Goh et al. 1992).

A pesar de que el inventario más reciente indica que en Costa Rica existen más de 1400 especies de orquídeas (Mora-Retana y García 1992), son muy pocos los estudios hechos sobre las relaciones micorrícicas en esta familia (Lesica y Antibus 1990, Richardson et al. 1993).

El propósito de este trabajo fue estudiar la presencia de micorrizas en orquídeas epífitas y terrestres, nativas de Costa Rica, establecidas en forma natural en los bosques secundarios del Jardín Botánico Lankester.

\section{MATERIALES Y METODOS}

Se estudiaron 24 especies de orquídeas, epífitas y terrestres, nativas de Costa Rica; 12 introducidas y 12 establecidas en forma natural en los bosques secundarios del Jardín Botánico Lankester, localizado en la provincia de Cartago a 1400 m.s.n.m., con una temperatura diaria promedio de $18-24^{\circ} \mathrm{C}$ y una precipitación anual de $1200 \mathrm{~mm}$. De cada especie se seleccionaron y marcaron de una a cinco plantas adultas distribuidas en diferentes partes del bosque. Luego se cortaron raíces de apariencia sana, de 2 a $5 \mathrm{~cm}$ de longitud, tomando como referencia el ápice, y que estuvieran en contacto con el substrato. En las especies Epidendrum radicans y Huntleya burtii, también se estudiaron muestras de raíces aéreas. La recolección se realizó durante todo el año, aunque las terrestres se recolectaron durante la estación lluviosa de julio a diciembre.

Las raíces se lavaron y se cortaron en segmentos de aproximadamente $1 \mathrm{~cm}$ de longitud, se digirieron en $\mathrm{KOH}$ al $10 \%(\mathrm{p} / \mathrm{v})$ por una hora a $90^{\circ} \mathrm{C}$. Posteriormente se decantaron y lavaron varias veces en agua destilada para luego neutralizarlas con ácido clorhídrico al $1 \%$ por una hora a $90^{\circ} \mathrm{C}$. Después de decantar y lavar en agua destilada. los tejidos se tiñeron con una solución de "azul directo" (Catálogo SIGMA, año 1996, producto 2535) en ácido láctico $(0.5 \mathrm{~g} / \mathrm{l})$ por una hora a $90^{\circ} \mathrm{C} \mathrm{y}$ se fijaron en glicerol. Se realizaron cortes longitudinales y se observaron al microscopio de luz para determinar la presencia de pelotones de hongos micorrícicos.

\section{RESULTADOS}

En todas las especies estudiadas se detectaron micorrizas en las células corticales de las raíces. Las hifas eran septadas y estaban organizadas en pelotones (Figs. 1, 2). En las orquídeas terrestres, las micorrizas se encontraron en todos los individuos examinados. En las epífitas se encontraron individuos sin micorrizas. No se observaron diferencias en la presencia de micorrizas entre especies introducidas o establecidas en forma natural (Cuadro 1). 


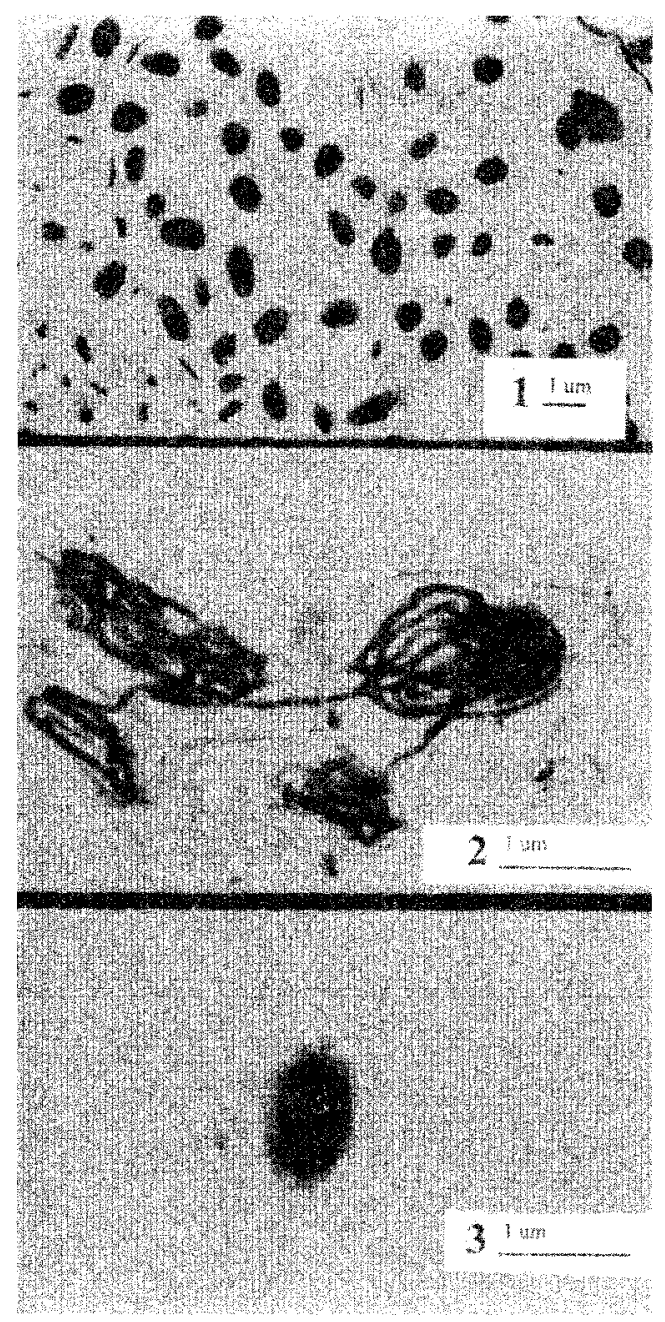

Fig. 1. Células radicales de Sarcoglottis sp. con pelotones. Fig. 2. Células radicales de Lockhartia amoena con hifas interconectadas en células vecinas.

Fig. 3. Células radicales de Sarcoglottis sp.con pelotones parcialmente digeridos.

En las especies epífitas Epidendrum radicans y Huntleya burtii, no se encontraron micorrizas en las raíces aéreas, únicamente en las que estaban en contacto con el substrato.

En todas las muestras de raíces en las que se detectaron micorrizas, la infección fue discontinua.
En las raíces de algunas especies la cantidad de células infectadas con hongos fue alta (Fig. 1). Se observaron hifas arregladas en pelotones en dos estados: uno en el que las hifas formaron ovillos y se pudieron distinguir las conexiones con las células adyacentes, además de los septos (Fig. 2), y otro con una masa amorfa de hifas en proceso de digestión (Fig. 3).

\section{DISCUSION}

El grado de infección micorrícica de las orquídeas costarricenses, epífitas o terrestres, establecidas en forma natural o introducidas en los bosques del Jardín Botánico Lankester es muy alto.

Esta observación contrasta con resultados obtenidos en estudios realizados en diversas áreas tropicales (Hadley y Williamson 1972, Hadley 1986, Lesica y Antibus 1990), incluyendo estudios realizados en los bosques lluviosos de Monteverde y la Selva en Costa Rica por Lesica y Antibus (1990) y por Richardson y colaboradores (1993) quienes encontraron incidencias de infección inferiores al 50\%. Por el contrario, nuestros resultados apoyan las investigaciones que sostienen que la incidencia de infección en las orquídeas terrestres y epífitas tropicales puede ser tan alta como la observada en orquídeas terrestres de climas templados (Benzing y Friedman 1981, Benzing 1982, Alexander 1987, Bermudes y Benzing 1989, Goh et al. 1992).

La ausencia de micorrizas en las raíces aéreas de dos especies así como la infección discontinua, también observada en otras especies (Hadley y Williamson 1972, Benzing 1982, Goh et al. 1992) refuerza la importancia del papel del substrato para el establecimiento de la infección micorrícica. En las orquídeas terrestres la infección en las nuevas raíces ocurre rápidamente mediante propágulos existentes en el suelo o por raíces infectadas o muertas el año anterior (Bridge 1979). En la mayoría de 


\section{CUADRO 1}

Presencia de micorrizas en orquídeas cultivadas en el Jardín Botánico Lankester

Especie

Cattleya skinneri Batem.

Cochleanthes discolor (Lindl.) Schultes \& Garay

Comparettia falcata Poepp. \& Endl.

Encyclia cordigera (H.B.K.) Dressler

Encyclia ochracea (Lindl.) Dressler

Epidendrum ciliare L.

Epidendrum radicans Pav. ex Lindl.

Gongora sp.

Govenia liliacea (Llave \& Lex.) Lindl.

Huntleya burtii Rchb.f.

Leochilus labiatus (Sw.) Ktze.

Lockhartia amoena Endres \& Rchb.f.

Malaxis sp.

Myrmecophila tibiscinis (Batem.) Rolfe

Oerstedella centradenia Rchb.f.

Oncidium sp.

Ornithocephalus sp.

Sobralia sp.

Sobralia sp.

Sobralia sp.

Sobralia sp.

Sarcoglottis sp.

Sarcoglottis sp.

Sarcoglottis sp.

$\begin{array}{cccc}\text { Hábito } & \text { Origen } & \mathrm{N}^{\circ} \text { individuos } & \mathrm{N}^{\circ} \text { individuos } \\ (*) & (* *) & \text { estudiados } & \text { con micorrizas }\end{array}$

* $\mathrm{E}=$ epífito, $\mathrm{T}=$ terrestre** $\mathrm{I}=$ introducida, $\mathrm{N}=$ establecida naturalmente.

$\begin{array}{rll}\text { I } & 5 & 1 \\ \mathrm{I} & 3 & 3 \\ \mathrm{~N} & 5 & 5 \\ \mathrm{I} & 5 & 4 \\ \mathrm{~N} & 5 & 4 \\ \mathrm{I} & 5 & 5 \\ \mathrm{~N} & 3 & 3 \\ \mathrm{I} & 4 & 4 \\ \mathrm{~N} & 4 & 4 \\ \mathrm{I} & 5 & 5 \\ \mathrm{~N} & 5 & 5 \\ \mathrm{I} & 5 & 4 \\ \mathrm{~N} & 2 & 2 \\ \mathrm{I} & 5 & 1 \\ \mathrm{~N} & 5 & 4 \\ \mathrm{~N} & 5 & 5 \\ \mathrm{~N} & 5 & 5 \\ \mathrm{I} & 1 & 1 \\ \mathrm{I} & 1 & 1 \\ \mathrm{I} & 1 & 1 \\ \mathrm{I} & 1 & 1 \\ \mathrm{~N} & 5 & 5 \\ \mathrm{~N} & 5 & 5 \\ \mathrm{~N} & 3 & 5\end{array}$

las epífitas la infección es necesaria para la germinación de las semillas; después de la diferenciación de los tejidos el hongo se restringe a la zona cortical e inicia el contacto con el substrato mediante hifas que salen de las raíces y absorben nutrimentos (Bridge 1979, Harley y Smith 1983).

La abundancia de micorrizas encontradas en las orquídeas nativas, epífitas y terrestres evaluadas en este estudio, contribuye a explicar la alta regeneración natural que se observa en los bosques secundarios del Jardín Botánico Lankester. Las semillas y plántulas de orquídeas son micótrofas obligadas que dependen del suministro de carbohidratos para germinar y crecer durante los primeros estadios (Arditti
1992). Las plantas adultas se pueden considerar micótrofas facultativas que se aprovechan de las hifas para obtener nutrientes en solución y compuestos orgánicos cuando digieren las hifas (Hadley y Williamson 1972, Benzing y Friedman 1981, Hadley 1982).

Dado que la infección micorrícica depende de la distribución y densidad de la flora fúngica en el ambiente, nuestros resultados sugieren que en los bosques naturales del Jardín Botánico Lankester existe una amplia distribución de los hongos asociados usualmente con la formación de micorrizas en la familia Orchidaceae, principalmente hongos imperfectos y derivados del complejo Rhizoctonia (Warcup y Talbot 1971, 1980, Warcup 1981, 1985). 


\section{AGRADECIMIENTOS}

Este estudio se desarrolló con la colaboración del Centro de Investigación General de la Universidad Estatal a Distancia, la Universidad de Costa Rica y el Museo Nacional de Costa Rica. Agradecemos el apoyo económico de la Universidad Estatal a Distancia y la Vicerrectoría de Investigación de la Universidad de Costa Rica, mediante el proyecto 814-96-557. Agradecemos a Jaime Aguilar por su ayuda técnica en el laboratorio y a Fabio Blanco por permitir el uso de equipo óptico.

\section{REFERENCIAS}

Alexander, C. E. 1987.'Mycorrhizal infection in adult orchids, p. 324-327. In D.M. Sylvia, I. Hung \& J.H. Graham (eds.). Mycorrhizae in the Next Decade, Practical Aplications and Research Priorities. University of Florida, Gainsville.

Arditti, J. 1992. Fundamentals of Orchid Biology. Wiley. Nueva York. 544 p.

Atwood, J.T. Jr. 1986. The size of the Orchidaceae and the systematic distribution of epiphytic orchids. Selbyana 9: 171-186.

Benzing, D.H. 1982. Mycorrhizal infections of epiphytic orchids in sourthen Florida. Amer. Orchid Soc. Bull. 51: 618-622.

Benzing, D. H. \& W.H. Friedman. 1981. Mycotrophy: its occurrence and possible significance among epiphyte Orchidaceae. Selbyana 5: 243-247.

Bermudes, D.\& D.H. Benzing. 1989. Fungi in neotropical epiphyte roots. BioSystems 23: 65-73.

Bridge,W.C. 1979. The ecology of fungy. CRC, Boca Raton, Florida, p.187-190.

Currah, R.S. 1991. Taxonomic and developmental aspects of the fungal endophytes of terrestrial orchid mycorrhizae. Lindleyana 6: 211-213.

Currah.R.S, L. Singl \& S. Hambleton. 1987. New records and taxa of fungi from the mycorrhizae of terrestrial orchids of Alberta. Canad. J.Bot. 65: 2473-2482.
Goh, C.J., A. A. Sim \& G. Lim. 1992. Mycorrhizal association in some tropical orchids. Lindleyana 7: 13-17.

Hadley, G. 1982. Orchid mycorrhizae. p. 83-118. In J. Arditti (ed.). Orchid Biology: reviews and perspectives II. Cornell University, Ithaca, Nueva York.

Hadley, G. 1985. Mycorrhiza in tropical orchids, p.154159. In A. N. Rao (ed). Proceedings of the 5th Asian Orchid Congress Seminar, Singapur., Parks and Recreation Department, Ministry of National Development. Singapur.

Hadley, G. 1986. Mycorrhiza in tropical orchids, p.154 159. In A. N. Rao (ed.). Proceeding of the 5th Asian Orchid Congress Seminar, Singapur. Park and Recreaion Deparment, Ministry of National Development, Singapur.

Hadley, G. \& B. Williamson. 1972. Features of mycorrhizal infection in some Malayan orchids. New Phytol. 71: 1111-1118.

Harley, J.L.\& S.E. Smith. 1983. Mycorrhizal symbiosis Academic, Londres, p.268-295.

Lesica, P. \& R. Antibus. 1990. The ocurrence of mycorrhizae in vascular epiphytes of two Costa Rican Rain Forest. Biotropica 22: 250-258.

Marchisio, V.F., G. Berta, A.Fontana \& F.M. Mannina 1985. Endophythes of wild orchids native to Italy: their morphology, caryology, ultrastructure and cytochemical characterization. New Phytol. 100: 623-64

Mora-Retana, D.E \& J.B.García. 1992. Lista actualizada de las orquídeas de Costa Rica. Brenesia 37: 79-124.

Ramsay, R.R., K. Sivasithamparam \& K.W. Dixon. 1987. Anastomosis groups among Rhizoctonia like endophytic fungi in southwestern Australian Pterostilis species (Orchidaceae). Lindleyana 2: 161-166.

Richardson, K.A., R.S. Currah \& S. Hambleton. 1993. Basidiomycetous endophytes from the roots of Neotropical epiphytic Orchidaceae. Lindleyana 8: 127-137.

Tsutsui, K. \& M. Tomita. 1986. Symbiotic germination of Spiranthes sinensis Ames associated with some orchid endophytes. J. Fac. Agric. Hokkaido Univ. 62: 440-452.

Uetake, Y., K. Kobayashi \& A. Ogoshi. 1992. Ultraestructural changes during the symbiotic development of Spiranthes sinensis (Orchidaceae) protocorms associated with binucleate Rhizoctonia anastomosis group C. Mycol. Res. 96: 199-209. 
Warcup, J.H. 1981. The mycorrhizal relationships of Australian orchids. New Phytol. 87: 371-381.

Warcup, J.H. 1985. Rhizanthella gardneri (Orchidaceae), its endophyte and close asociation with Melalenca uncinata (Myrtaceae) in Western Australia. New Phhytol. 70: 99-273-280.
Warcup, J.H. \& A. Talbot. 1967. Perfect states of Rhizoctonias associated with orchids. New Phytol. 66: 631

Warcup, J.H. \& A. Talbot. 1971. Perfect states of Rhizoctonias associated with orchids II. New Phytol. 70: 35-40.

Warcup, J.H. \& A. Talbot. 1980. Perfect estates of Rhizoctonias associated with orchids.III. New Phytol. 86: 267-272. 